\title{
Aproximações da produção científica em ciências da saúde na ciência da informação no Brasil
}

\author{
Approximations of scientific production in health sciences in the \\ information science in Brazil
}

\section{Aproximaciones de la producción científica en ciencias de la salud en la ciencia de la información en Brasil}

\author{
Rene Faustino Gabriel Junior ${ }^{1, a}$ \\ rene.gabriel@ufrgs.br | https://orcid.org/oooo-0003-1021-3360
}

Fernanda Bochi $i^{2, b}$

nandabochi@gmail.com | https://orcid.org/oooo-0002-3275-0725

Ana Maria Mielniczuk de Moura ${ }^{1, c}$

ana.moura@ufrgs.br | https://orcid.org/oooo-0002-7811-4660

\footnotetext{
${ }^{1}$ Universidade Federal do Rio Grande do Sul, Programa de Pós-Graduação em Ciência da Informação. Porto Alegre, RS, Brasil.

2 Universidade Estadual Paulista, Programa de Pós-Graduação em Ciência da Informação. Marília, SP, Brasil.

a Doutorado em Ciência da Informação pela Universidade Estadual Paulista.

${ }^{\text {b } M e s t r a d o ~ e m ~ C o m u n i c a c ̧ a ̃ o ~ e ~ I n f o r m a c ̧ a ̃ o ~ p e l a ~ U n i v e r s i d a d e ~ F e d e r a l ~ d o ~ R i o ~ G r a n d e ~ d o ~ S u l . ~}$

'Doutorado em Comunicação e Informação pela Universidade Federal do Rio Grande do Sul.
}

\section{RESUMO}

O trabalho apresentado neste artigo analisou as aproximações da produção científica em ciências da saúde na área de ciência da informação, no Brasil, com base nos artigos indexados na Base de Dados Referenciais de Artigos de Periódicos em Ciência da Informação (Brapci), no período de 1972 a 2020. A partir da coleta pelo termo 'saúde', os dados foram organizados a fim de: identificar os autores mais produtivos; descrever as formas de coautoria; investigar as características das revistas utilizadas para publicação; analisar as temáticas identificadas a partir das palavras-chave. Foram coletados 1.293 documentos, dos quais, após limpeza, totalizaram 1.140 registros, que foram administrados no software VOSviewer. Verificou-se que $3,3 \%$ dos documentos em ciência da informação indexados na base abordam alguma questão da área de saúde. A Revista Eletrônica de Comunicação, Informação e Inovação em Saúde (Reciis) publicou 43,2\% de todos os trabalhos recuperados e uma mostrou prevalência de autoria múltipla.

Palavras-chave: Ciência da informação; Ciências da saúde; Produção científica; Colaboração científica; Estudos métricos da informação.

\section{ABSTRACT}

The work presented in this article analyzed the approximations of scientific production in health sciences in the area of information science in Brazil based on articles indexed by the Base de Dados Referenciais de Artigos de Periódicos em Ciência da Informação (Brapci) from 1972 to 2020. After searching the term 'health', the data were organized to in order to identify the most productive authors; to describe the forms of coauthorship; to investigate the characteristics of the journals used for publication; and to analyze the 
themes identified by means of the keywords. 1,293 documents were collected and after cleaning the total came to 1,140 records that were visualized using the VOSviewer software. It was verified that $3.3 \%$ of the documents concerning information science which was indexed in the database address some issue of health field. The Reciis - Revista Eletrônica de Comunicação, Informação e Inovação em Saúde (Electronic journal of communication, information and innovation in health) published $43.2 \%$ of all works obtained and has revealed a prevalence of multiple authorship.

Keywords: Information science; Health sciences; Scientific production; Scientific collaboration; Metric information studies.

\section{RESUMEN}

El trabajo presentado analizó las aproximaciones de la producción científica en ciencias de la salud en el área de ciencias de la información en Brasil con base en artículos indexados en la Base de Dados Referenciais de Artigos de Periódicos em Ciência da Informação (Brapci) en el período de 1972 a 2020. A partir de la recolección del término 'salud', los datos fueron organizados para identificar a los autores más productivos; describir las formas de coautoría; investigar las características de las revistas utilizadas para la publicación; analizar los temas identificados a partir de las palabras clave. Han sido recopilados 1.293 documentos y después de la limpieza totalizaron 1.140 registros que han sido visualizados en el software VOSviewer. Se han verificado que $3.3 \%$ de los documentos conciernentes a la ciencia de la información indexados en la base de datos abordan algún tema del área de salud. La Reciis - Revista Eletrônica de Comunicação, Informação e Inovação em Saúde (Revista electrónica de comunicación, información y innovación en salud) publicó $43,2 \%$ del total de trabajos recuperados y ha evidenciado un predominio de autoría múltiple.

Palabras clave: Ciencia de la información; Ciencias de la salud; Producción científica; Colaboración científica; Estudios métricos de información.

Este artigo compõe o dossiê Estudos métricos da informação científica em saúde, parte 1.

Contribuição dos autores:

Concepção e desenho do estudo: Rene Faustino Gabriel Junior.

Aquisição, análise ou interpretação dos dados: Ana Maria Mielniczuk De Moura; Fernanda Bochi; Rene Faustino Gabriel Junior.

Redação do manuscrito: Ana Maria Mielniczuk De Moura; Fernanda Bochi; Rene Faustino Gabriel Junior.

Revisão crítica do conteúdo intelectual: Ana Maria Mielniczuk De Moura; Fernanda Bochi; Rene Faustino Gabriel Junior.

Declaração de conflito de interesses: não há.

Fontes de financiamento: Coordenação de Aperfeiçoamento de Pessoal de Nível Superior (Capes).

Considerações éticas: não há.

Agradecimentos/Contribuições adicionais: O presente trabalho foi realizado com apoio da Coordenação de Aperfeiçoamento de Pessoal de Nível Superior - Brasil (Capes) - Código de Financiamento 001.

Histórico do artigo: submetido: 19 maio 2021 | aceito: 25 ago. 2021 | publicado: 10 nov. 2021.

Apresentação anterior: não há.

Licença CC BY-NC atribuição não comercial. Com essa licença é permitido acessar, baixar (download), copiar, imprimir, compartilhar, reutilizar e distribuir os artigos, desde que para uso não comercial e com a citação da fonte, conferindo os devidos créditos de autoria e menção à Reciis. Nesses casos, nenhuma permissão é necessária por parte dos autores ou dos editores. 


\section{INTRODUÇÃO}

Dialogar sobre saúde no contexto da ciência da informação (CI) suscita uma série de reflexões importantes, uma vez que tanto as ciências sociais como a CI são áreas que buscam atender a necessidades sociais. Cientes da relevância da democratização do acesso e do uso das informações científicas em saúde, o Ministério da Saúde (MS), a Organização Pan-Americana da Saúde (Opas), o Escritório Regional da Organização Mundial da Saúde (OMS) e demais instituições assinaram um importante convênio que promoveu e expandiu as informações técnico-científicas das ciências da saúde. Nasceu, em 1967, a Biblioteca Regional de Medicina (Bireme), atendendo às necessidades informacionais de profissionais, pesquisadores e estudantes da saúde em toda a América Latina e no Caribe. Em 1982, a fim de manifestar sua função da melhor forma, passou a ser chamada de Centro Latino-Americano e do Caribe de Informação em Ciências da Saúde. A Bireme se tornou uma referência em informação científica e técnica em saúde, promovendo atividades de gestão de informação e conhecimento científico com o intuito de ampliar o fluxo de informação na área (BIREME, 2017).

Nesse processo da democratização do acesso à informação das publicações em saúde, foi criado, em 1992, o Congresso Regional de Informação em Ciências da Saúde (Crics), com a intenção de ser um congresso inovador no campo da informação e comunicação científica em saúde. Com mais de uma década de existência, o Seminário Internacional de Informação para a Saúde tornou-se um evento importante, promovendo interação e interlocução entre profissionais, pesquisadores e estudantes das áreas de CI, biblioteconomia, ciências da saúde, computação, gestão, ética e diversas outras, para discussão de temas relacionados à informação para a saúde. Isso permite compreender que existe uma consolidada e crescente colaboração entre as áreas. No Brasil, de acordo com as áreas de avaliação da Capes (2017), as ciências da saúde estão subdivididas em medicina, odontologia, farmácia, enfermagem, nutrição, saúde coletiva, fonoaudiologia, fisioterapia e terapia ocupacional e educação física. Essas áreas de conhecimento são segmentos que devem ser mais bem explorados pelos profissionais e pesquisadores da área de CI, uma vez que sua interdisciplinaridade possibilita o seu envolvimento com os diversos campos do saber.

Beraquet e Ciol (2010) observaram que profissionais da CI, como os bibliotecários, ainda apresentavam uma visão limitada sobre o campo de atuação, permanecendo atrelados à ideia de que só podem atuar em ambientes convencionais. As autoras apresentaram uma reflexão sobre o campo de atuação do profissional em ambientes não tradicionais, como a área da saúde, contribuindo com o entendimento das competências e habilidades desse profissional, para atuarem nas diversas áreas das ciências da saúde.

Prudêncio e Biolchini (2017) identificaram que a temática informação e saúde ainda é incipiente, sendo pouco abordada nos cursos de pós-graduação em CI. No entanto, destaca-se que na Associação Nacional de Pesquisa e Pós-Graduação em Ciência da Informação (Ancib), por exemplo, a partir da moção proposta, em 2010, pelo Instituto de Comunicação e Informação Científica e Tecnológica em Saúde (Icict), desde 2011, foi incluído no Encontro Nacional de Pesquisa da Ancib (Enancib) o GT 11 - Informação e Saúde, que realiza estudos de teorias, métodos, estruturas e processos informacionais em diferentes contextos da saúde, considerada em sua abrangência e complexidade. Ele trata do impacto da informação, das tecnologias, da inovação em saúde, da informação nas organizações de saúde, da informação, da saúde e sociedade e das políticas de informação em saúde, assim como da formação e capacitação em informação em saúde (FIOCRUZ, 2013). Contudo, observa-se que, mesmo antes da criação do GT11, a Revista Eletrônica de Comunicação, Informação e Inovação em Saúde (Reciis) já dava importante contribuição para a ampliação dos estudos em ciências da saúde no âmbito da CI. Esse periódico de acesso aberto publica artigos de interesse para as áreas de comunicação científica, informação e saúde e vem contribuindo para o aumento do fluxo de informação sobre a temática, sendo coordenado e editado pelo Icict da Fundação Oswaldo Cruz (Fiocruz). 
Esse GT surgiu no evento realizado em 2011, e as pesquisas nele apresentadas são dedicadas a aspectos da informação em saúde e que estavam sendo discutidas em outros grupos de trabalho. Com a criação desse GT, que tem como proposta fomentar ainda mais os diálogos das pesquisas sobre teorias, métodos e estruturas informacionais nos mais diversos contextos da saúde, analisa-se o impacto da informação, das tecnologias e das inovações em saúde, inclusive nas organizações de saúde, além de questões mais amplas de informação, saúde e sociedade e as políticas informacionais em saúde (GARCIA; ARAUJO; SOUZA, 2019).

A criação desse GT demonstra a consolidação das pesquisas na área da saúde no âmbito da CI, o que justifica investigar as aproximações da produção científica dessas áreas, objeto do estudo ora apresentado. O foco de análise do trabalho são os artigos indexados na Brapci, com o objetivo geral de analisar as aproximações da produção científica em ciências da saúde na área de CI, no Brasil. Como objetivos específicos, tem-se: identificar os autores mais produtivos na temática; descrever as formas de coautoria; investigar as características das revistas utilizadas para publicação; analisar as temáticas identificadas a partir das palavras-chave (assuntos).

\section{PRODUÇÃO CIENTÍFICA E COLABORAÇÃO CIENTÍFICA}

O desenvolvimento científico está fundamentado na premissa da comunicação, segundo a qual ela obtém informação e transmite também por meio da produção científica. A comunicação científica proporciona aos pesquisadores o intercâmbio de ideias e informações, de modo que tal atividade entra num fluxo helicoidal, como menciona Guimarães (2017). Como afirma Targino (2009), tal prática reitera o quanto a comunicação científica se fundamenta na informação e produção científica. A produção científica, segundo Lourenço (1997), está relacionada à produção documental sobre determinado assunto de interesse de uma comunidade científica, independentemente do suporte em que esteja, papel ou eletrônico. Para Botomé (1996), a produção científica só tem sentido se existir um destino para o que for produzido e consumido. Nessa linha, Meadows (1999) destaca que a produtividade científica tem importância na consolidação de novos conhecimentos em diferentes áreas de conhecimento. No entanto, para Targino (2009), a comunicação científica e todo o processo que envolve a divulgação da ciência têm como intuito mostrar a força da divulgação científica, principalmente, nas pesquisas envolvendo saúde.

Nascimento e coautores (2021) constataram que o total da produção científica brasileira cresceu a uma taxa exponencial anual de 6,61\% entre os anos de 1990 e 2017, observando, porém, uma desaceleração do crescimento dessa produção nas áreas avaliadas na primeira metade da década de 2000. Os autores afirmam que a produção científica nacional registrou um período de crescimento acelerado entre 1990 e os primeiros anos da década de 2000, com taxas exponenciais anuais de até 17,6\% (ciências sociais aplicadas) e dobrando de tamanho a cada quatro anos. Outro dado interessante apontado pelos autores é que a produção científica brasileira anual saltou de, aproximadamente, 40 mil artigos, em 1990, para mais de 391 mil, em 2016, maior valor da série. Nesse período, o número de artigos publicados por milhão de habitantes no país cresceu, aproximadamente, 500\%, saindo de 340 publicações, em 1990, para mais de 1.800 , em 2017.

Sidone, Haddad e Mena-Chalco (2016) apontam para uma desaceleração da produção nos últimos anos nas grandes áreas de ciências da saúde e ciências iológicas, fato também indicado por Nascimento e coautores (2021), ao constatarem que essas áreas registraram as menores taxas de crescimento, 5,87\% (ciências biológicas) e 5,19\% (ciências da saúde), no comparativo com as outras áreas do conhecimento.

Mueller (2005) destaca que diversos aspectos específicos das grandes áreas podem ter influenciado o desempenho da produção científica recente, como a intensidade de programas de financiamento, o número de periódicos, a quantidade de cursos de graduação e pós-graduação, o número de docentes, pesquisadores e discentes, as metodologias de pesquisa, entre outros. 
De acordo com Bicalho (2011), a CI tem sua origem interdisciplinar, trabalhando com a produção do conhecimento científico em todas as áreas do conhecimento. A autora destaca que, para o desenvolvimento da complexidade de seu objeto e dos problemas que deve tratar, a CI se apropriou de diversas teorias e metodologias de outras disciplinas, bem como da contribuição e participação de pesquisadores de diversas áreas do conhecimento. Porém, em seus resultados, não foi possível identificar a característica interdisciplinar da área. Em outro estudo, Smit (2002) destaca que existe uma aparente discrepância entre o que é dito (interdisciplinaridade da área) e o que é feito, existindo um isolamento da pesquisa em CI do ambiente de pesquisa de outras disciplinas. Em estudo mais recente, Moreira, Valentim e Santana (2018), ao realizar estudo sobre interdisciplinaridade da CI e suas contribuições para o estudo do compartilhamento de dados governamentais na internet, identificaram que existe uma aproximação na formação de mestres e doutores nos programas de pós-graduação (PPG) em CI oriundos de outras áreas do conhecimento, reforçando a característica interdisciplinar.

Nesse contexto do processo de construção da produção científica, os pesquisadores compartilham objetivos e metas em comum, dependendo, algumas vezes, da participação efetiva das diferentes comunidades epistêmicas para o desenvolvimento de novos saberes. Essa participação e troca de conhecimento fazem crescer a busca de colaboração e o interesse por ela entre pesquisadores que atuam em uma mesma instituição ou não e, até mesmo, em áreas semelhantes ou distintas. Os primeiros estudos sobre colaboração científica datam do final da década de 1950 (SONNENWALD, 2008). Como definem Katz e Martin (1997), a colaboração científica ocorre quando dois cientistas compartilham dados, equipamentos ou ideias em um projeto, que resulta, geralmente, em experimentos e análises de pesquisa publicados em um artigo. Hilário, Grácio e Guimarães (2018) consideram que a colaboração é uma atividade adotada pelos pesquisadores como estratégia para viabilizar e potencializar a pesquisa. Para eles, a coautoria, um dos objetos deste estudo, se caracteriza como um item da colaboração científica, apresentando benefícios cognitivos para os pesquisadores envolvidos nessa prática colaborativa. No entanto, Hilário e Lazzaroto (2020) salientam que o indivíduo declarado como autor deve ter participação efetiva no processo de construção do conhecimento científico, apresentando contribuições substanciais para o desenvolvimento do trabalho, fato que, em alguns momentos, não ocorre no processo colaborativo, que, muitas vezes, está voltado para o abastecimento de uma carência de infraestrutura e recursos materiais. Porém, observa-se que, neste estudo, a coautoria entra como um produto da colaboração, conforme elucidado por Hilário, Grácio e Guimarães (2018).

Vanz e Stumpf (2010), em seu trabalho de revisão sobre o assunto, concluem que a colaboração científica é um fenômeno antigo, que vem crescendo em todas as áreas da ciência e em todos os países, mostrando-se uma prática saudável e que beneficia não só a comunidade científica, como também as instituições e os países aos quais os pesquisadores estão vinculados. $\mathrm{O}$ acesso a equipamentos e materiais, o compartilhamento de conhecimento científico, a maior especialização e o aprofundamento das pesquisas são apenas alguns dos benefícios gerados pelas colaborações. E, para Glanzel (2001), o trabalho escrito em coautoria, uma das facetas da colaboração, apresenta maior probabilidade de aceite e maior número de citações, quando comparado a trabalhos publicados individualmente.

Embora autoras como Hilário e Lazzarotto (2020) apresentem conceitos profundos sobre colaboração e aspectos éticos, este estudo buscou elucidar a importância da prática colaborativa para o desenvolvimento da informação em saúde, uma vez que a colaboração na produção de conhecimento tem se mantido num patamar alto nas diversas áreas do saber, tanto em âmbito nacional como internacional. Em relação ao total de colaborações científicas domésticas, ou seja, entre pesquisadores brasileiros, esse número cresceu de maneira bastante acelerada, saltando de cerca de 500 mil, no triênio 1992-1994, para cerca de 13 milhões, em 2007-2009 (SIDONE, HADDAD, MENA-CHALCO, 2016). Isso evidencia o papel 
fundamental desempenhado pela colaboração doméstica na evolução recente da produção científica brasileira. Semelhantemente ao padrão verificado na evolução da produção acadêmica, nota-se uma intensa desaceleração do aumento no total de colaborações, uma vez que a taxa de crescimento de $146 \%$, observada entre os períodos de 1995-1997 e 1998-2000, reduziu-se para cerca de 40\%, entre os últimos triênios, percentual que ainda permanece em patamar elevado.

$\mathrm{Na}$ área de saúde, a prática da colaboração apresenta-se como fundamental e vem crescendo ao longo dos anos, tornando-se predominante nas publicações da área (SOBRAL et al., 2016). Entre os anos de 1998 e 2000, os artigos de autoria única eram a maioria, sendo mais de $60 \%$ do quantitativo total. Entre $2004 \mathrm{e}$ 2006, o gráfico inverteu, e os artigos em colaboração já representavam cerca de $80 \%$ daqueles produzidos na área de saúde. Os autores também destacam que as motivações mais importantes dos pesquisadores em defesa da colaboração são: a possibilidade de enfrentar grandes problemas de pesquisa; a obtenção e/ ou ampliação de fomento; o processo de validação dos trabalhos realizados em grupo; e a possibilidade de avaliar previamente os produtos de pesquisa em relação às instâncias avaliadoras.

Bettio, Alvarez e Vanz (2017) identificaram, no estudo sobre a produção e colaboração científica da Universidade Federal de Ciências da Saúde de Porto Alegre (UFCSPA), que a autoria múltipla se mostra predominante (98,6\%). Desses trabalhos, $17,7 \%$ se realizaram em coautoria internacional. Estados Unidos, Reino Unido, Canadá, Espanha, França e Argentina são os principais parceiros. Dos 1.905 documentos com colaboração, 86,8\% contam com duas ou mais instituições coautoras, com predomínio de universidades nacionais públicas, além de instituições hospitalares. Também na área da saúde, Maia (2006) investigou as características de colaboração dos professores do Programa de Pós-graduação em Epidemiologia da Universidade Federal de Pelotas, constatando que os professores publicam mais artigos em autoria compartilhada (93\%) do que individuais. Contudo, como bem elucida Queiroz (2020), é importante salientar que a autoria múltipla, embora ocorra com frequência, não pode ser considerada uma prática unânime de todas as áreas do conhecimento.

Diante do que foi exposto, compreende-se que existe uma sinergia muito forte entre a CI e as ciências da saúde, uma vez que ambas proporcionam recursos fundamentais para assegurar a qualidade de vida e o bem-estar humano. A primeira trata, organiza e comunica o que a segunda aplica, permitindo que o fluxo de informação e as colaborações entre pesquisadores ou instituições ocorram. Fato elucidado por Targino (2009), que considera a saúde, bem como a informação em saúde recursos básicos e fundamentais para elevar a qualidade de vida dos cidadãos de uma nação. Além disso, observa-se que estudos métricos, como os apresentados por Lazzarotto (2017), Grácio e Oliveira (2012) e Mattos e Job (2008), estão sendo relacionados às pesquisas em saúde, o que permite identificar de forma quantitativa o que está sendo desenvolvido sobre CI e informação e saúde, oportunizando projetar estudos futuros.

\section{METODOLOGIA}

Para identificar a presença da área de saúde nas publicações brasileiras de CI, partiu-se de uma busca na Base de Dados Referenciais de Artigos de Periódicos em Ciência da Informação (Brapci), utilizando o termo 'saúde' nos campos, no título, nas palavras-chave e no resumo, tendo como delimitação temporal os anos de 1972 até 2020 e publicações em revistas de ciências da informação brasileiras. Justifica-se a definição do início em 1972 por ser o ano da primeira publicação das três primeiras revistas especializadas em CI. De forma a restringir o recorte, optou-se por utilizar somente o termo 'saúde', garantindo desta forma maior proximidade com a proposta do estudo, e não buscando todas a variações possíveis das áreas subordinadas. A Brapci foi escolhida para a pesquisa por indexar 70 revistas brasileiras, 16 revistas estrangeiras, e os eventos do Encontro Nacional de Pesquisa e Pós-graduação em Ciência da Informação (Enancib), Encontro Brasileiro de Bibliometria e Cientometria (EBBC), Congresso de Gestão Estratégica 
da Informação e a Sociedade Brasileira para Organização do Conhecimento ISKO-Brasil (a sigla ISKO corresponde a International Society for Knowledge Organization).

O resultado da busca possibilitou a identificação de 1.293 documentos. Com o intuito de delimitar para as revistas brasileiras, foi aplicado um filtro removendo as revistas estrangeiras e os eventos, resultando em um corpus de 1.140 registros. Considerando que a base tem indexados 39.343, isso significa que apenas 3,3\% dos documentos em CI abordam alguma questão da área de saúde. Do total de trabalhos, foram identificados 2.263 autores, sendo que, destes, 1.902 (84,0\%) tiveram apenas uma publicação, e 224 (9,9\%) duas publicações. Com mais de duas publicações no período analisado, foram identificados 137 $(6,1 \%)$ autores do total do corpus. Para gerar a correlação das palavras-chave, foram extraídos os termos de todos os trabalhos recuperados e excluídos os termos 'ciência da informação' em outros idiomas, diferentes do português, por estarem presentes na maioria dos artigos. Ainda para padronizar, foram retirados os acentos dos termos. Com a aplicação desses filtros, foi criada a tabela de correção, utilizando as ferramentas bibliográficas disponíveis na Brapci (ARBOIT; BUFREM; LAZZAROTTO, 2010), e os termos enviados para o VOSviewer (CWTS, 2021) para criação do gráfico de aproximações temáticas. De modo a diminuir a poluição visual, foram definidos, na apresentação, os termos (nodes) que tivessem, no mínimo, 20 relações ( $\operatorname{arcs}$ ), e foi utilizada a aproximação de 10 e repulsão de 1, no modo fracionado. A escolha do uso da Brapci deu-se, sobretudo, por ser uma base de dados nacional da CI que indexa tudo o que é produzido na área, além de ser de fácil acesso e uso, mantendo-se atualizada e apresentando conteúdo de acesso aberto e possibilidade de acesso aos metadados.

\section{RESULTADOS E ANÁLISES}

Ao analisar o corpus constituído de 1.140 documentos, quanto ao tipo de autoria, pode-se observar que, do total de publicações, 292 (25,6\%) foram de autoria única, e 848 (74,4\%) de autoria múltipla, distribuídas em 367 (32,2\%) em dupla autoria, 231 (20,3\%) em autoria tripla, 124 (10,9\%) em autoria quádrupla, 126 $(11,1 \%)$ com cinco ou mais autores. Um estudo realizado por Mattos e Job (2008) reitera os resultados deste estudo, pois aponta que, dos 34 artigos de autores brasileiros publicados na Scientometrics, no período de 1978 até 2006, nove apresentam autoria única, um tem cinco autores, quatro são de quatro autores, 10 artigos foram escritos por dois, e 10 por três autores, indicando uma preferência por autoria compartilhada.

A influência de poucas colaborações e coautorias na área de CI, no Brasil, é evidenciada pela pesquisa realizada por Mueller e Pecegueiro (2001), com base na revista Ciência da Informação, entre os anos de 1990 e 1999. Eles constataram que $78,23 \%$ dos artigos foram assinados por um único autor. Resultado semelhante foi obtido por Bufrem e outros autores (2010), que analisaram 28 revistas de CI brasileiras, entre 1970 e 2008, obtendo um indicador de 68,1\% de autoria única. Em uma análise diacrônica, Gabriel Junior (2017) analisou os tipos de autorias de 1972 até 2016 de forma cronológica, o que possibilitou identificar que as autorias únicas vêm diminuindo ao longo do tempo, com um forte crescimento a partir de 1994 da dupla autoria, que, em 2008, sobrepujou a autoria única. Entretanto, a média de autores por documentos não passou de 1,8 autores por trabalho.

A média de coautores por trabalhos, nesta pesquisa, foi de 1,98 autores por documento. A Figura 1 destaca os oito autores mais produtivos, com artigos publicados nas revistas de CI brasileiras, com 10 ou mais publicações. Neves e Ferreira (2014), que analisaram as publicações de autores portugueses no repositório internacional e-Prints in Library and Information Science (e-LIS), observaram que 67\% dos trabalhos eram de autoria única.

Vanz (2009) identificou que existe uma diferenciação do número de autores por trabalho nas diversas áreas do conhecimento e que a física tem o maior número de coautores (13,3 por artigo), enquanto as áreas de saúde têm 4,6 a 7 autores por trabalho. Dessa forma, pode-se observar que o número de autores nas 
áreas de saúde é muito superior ao da área de CI, e que a aproximação da CI com essas áreas aumentou o número de coautores neste estudo. Uma análise realizada, na Reciis, por Bochner e outros autores (2014), identificou que a média de autores da CI, em 2013, era de 3,43, ou seja, houve um impacto direto da cultura dos pesquisadores da área de saúde.

A Figura 1, a seguir, apresenta a série histórica das publicações dos autores que tiveram mais de 10 publicações no período e corpus analisado.

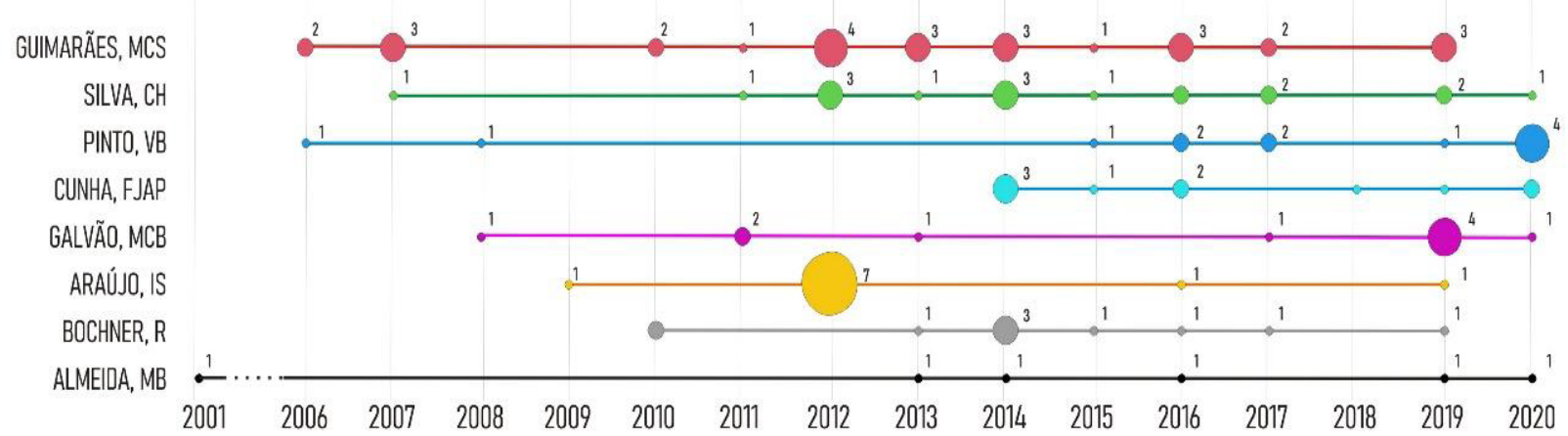

Figura 1 - Autores com dez ou mais publicações na área de saúde nos artigos em Cl, no Brasil Fonte: elaborado pelos autores.

Dos autores enfatizados, destaca-se Maria Cristina Soares Guimarães, a mais produtiva, com 27 publicações, entre 2006 e 2019. A autora tem formação, na graduação, em engenharia química e mestrado e doutorado em ciência da informação pela UFRJ/Ibict. É pesquisadora da Fundação Oswaldo Cruz e professora permanente do Programa de Pós-Graduação em Informação e Comunicação em Saúde do Icict (PPGICS/Icict). Guimarães foi a autora mais constante em suas publicações, com a identificação do primeiro trabalho, em 2006, e mantém as publicações até 2019.

Cícera Henrique Silva tem graduação em biblioteconomia pela UniRio, mestrado pela UFRJ/Ibict e doutorado pela Université dAix-Marseille II. Atualmente, é pesquisadora colaboradora do Instituto de Comunicação e Informação Científica e Tecnológica em Saúde - Icict, desde 2005, é docente do curso de especialização lato sensu Informação Científica e Tecnológica em Saúde e, desde 2009, professora permanente do Programa de Pós-Graduação em Informação e Comunicação em Saúde - PPGICS/Icict. Foram identificados 17 trabalhos publicados, sendo que 11 deles em coautoria com Maria C. S. Guimarães, relacionados ao acesso aberto, acesso livre e repositório institucional, principalmente envolvendo o Repositório Institucional da Fiocruz (Arca).

Com 12 trabalhos, destaca-se a professora do Departamento de Ciências da Informação da Universidade Federal do Ceará, Virgínia Bentes Pinto. Entre seus estudos na área de saúde, publicou sobre: uso de prontuários eletrônicos; representação e indexação de imagens; e linguagem documentária na área de saúde. Na Figura 1, pode-se observar que a autora iniciou o interesse pelo tema em 2006 e 2008, com uma publicação, retomando a temática a partir de 2015, com estudos do uso da instrumentalização da biblioteconomia e CI aplicados na saúde.

Francisco José Aragão Pedroza Cunha, professor da Universidade Federal da Bahia (UFBA), vinculado ao Instituto de Ciência da Informação, pontua com 10 trabalhos. Tem graduação em administração de empresas, mestrado em CI pela UFBA e doutorado em difusão do conhecimento, com trabalhos publicados na temática de mecanismos de difusão de conhecimentos gerenciais entre os sistemas e redes de atenção à saúde; gestão de documentos; e bases para a aprendizagem e a inovação organizacional em hospitais. Suas publicações têm início em 2014, sendo o pesquisador, entre os mais produtivos, o mais contemporâneo. 
Maria Cristiane Barbosa Galvão, com 10 trabalhos na área, é professora doutora do Departamento de Medicina Social da Faculdade de Medicina de Ribeirão Preto da Universidade de São Paulo. Bacharel em biblioteconomia e documentação pela Universidade de São Paulo (1992), realizou mestrado em comunicação pela Universidade de São Paulo (1997) e doutorado em ciência da informação pela Universidade de Brasília (2003), período em que realizou estágio na Universidade de Montreal (2002-2003).

Ressaltando o domínio do Icict da Fiocruz, destaca-se também, com 10 produções, Inesita Soares Araújo, graduada em comunicação social pela Universidade Federal de Pernambuco (UFPE), mestre e doutora em comunicação e cultura pela Universidade Federal do Rio de Janeiro (UFRJ). Atua como pesquisadora no Laboratório de Comunicação e Saúde do Instituto de Comunicação e Informação Científica e Tecnológica em Saúde (Laces/Icict) e docente permanente no Programa de Pós-Graduação em Informação e Comunicação em Saúde (PPGICS). Em 2012, publicou sete trabalhos com a temática relacionada à saúde.

Rosany Bochner, também do Icict, com oito publicações, é graduada em estatística, mestre em engenharia de produção na área de pesquisa operacional pela UFRJ e doutora em saúde pública pela Escola Nacional de Saúde Pública Sérgio Arouca da Fundação Oswaldo Cruz (Ensp/Fiocruz). Pesquisa na área de probabilidade e estatística, com ênfase em análise de dados na área da saúde, além de ter participado do desenvolvimento de sistemas de informação em saúde e na elaboração de materiais educativos e de divulgação científica. Atua como pesquisadora do Laboratório de Informação Científica e Tecnológica em Saúde do Instituto de Comunicação e Informação Científica e Tecnológica em Saúde (Licts/Icict), coordena o Sistema Nacional de Informações Tóxico-Farmacológicas (Sinitox) e é membro permanente do corpo docente do Programa de Pós-Graduação em Informação e Comunicação em Saúde do Icict (PPGICS).

Maurício Barcellos Almeida é professor do Departamento de Teoria e Gestão da Informação na Universidade Federal de Minas Gerais (UFMG), graduado em engenharia civil, mestre e doutor em ciência da informação pela UFMG. Pesquisa e atua, principalmente, com a temática de ontologias, representação do conhecimento e uso da terminologia nas práticas clínicas e em saúde, com cinco publicações na área.

Esses resultados apontam uma crescente no que tange à atuação do cientista da informação nos estudos voltados para as ciências da saúde. que não pode ser observado no estudo de Mattos e Job (2008), visto que esses autores identificaram, no período analisado, que os pesquisadores mais produtivos não eram cientistas da informação, mas tinham suas formações nas áreas de ciências biológicas, epidemiologia e demais da saúde. No entanto, o estudo realizado por Prudencio e Biolchini (2018), em que analisaram os currículos dos cursos de biblioteconomia presenciais e a distância, mostrou uma tímida, porém crescente, abordagem das temáticas informação em saúde nas disciplinas de graduação.

Na identificação das revistas mais produtivas, os artigos recuperados estavam distribuídos em 56 revistas brasileiras da área de CI, sendo que 17 delas concentram mais de 80\% das publicações, concentrando as publicações em revistas B1 que, de acordo com o Qualis Capes, são periódicos de excelência nacional, predominantes na área. Isso demonstrou uma concentração em poucos títulos de revistas. A Tabela $1 \mathrm{e}$ a Figura 2 ilustram os principais periódicos identificados na pesquisa em pauta. No estudo abordando os estudos métricos da informação como interdomínio entre CI e medicina, Lazzarotto (2017) identificou que, entre os 10 periódicos mais referenciados, seis são das ciências da saúde, dois são especificamente da CI sendo um internacional (Journal of the Association for Information Science and Technologie - JASIST), um nacional (CI) - e os demais apresentam um caráter mais interdisciplinar. Tal resultado é interessante, visto que a Revista Ciência da Informação é a mais antiga da área no Brasil. 


\section{Tabela 1 - Periódicos com mais de vinte publicações na temática}

\begin{tabular}{clccc} 
\# & Revista & $\begin{array}{c}\text { Total de } \\
\text { docs }\end{array}$ & $\begin{array}{c}\text { Qualis } \\
\mathbf{2 0 1 3} \\
\mathbf{2 0 1 6}\end{array}$ & $\begin{array}{c}\text { SCImago } \\
\text { Scopus }\end{array}$ \\
\hline 1 & Revista Eletrônica de Comunicação, Informação e Inovação em Saúde & 492 & $\mathrm{~B} 1$ & - \\
2 & Ciência da Informação & 52 & $\mathrm{~B} 1$ & $\mathrm{Q} 4$ \\
3 & Revista Fontes Documentais & 50 & - & - \\
4 & Revista Brasileira de Biblioteconomia e Documentação & 38 & $\mathrm{~B} 1$ & - \\
5 & Perspectivas em Ciência da Informação & 32 & $\mathrm{~A} 1$ & $\mathrm{Q} 2$ \\
6 & Tendências da Pesquisa Brasileira em Ciência da Informação & 31 & $\mathrm{~B} 1$ & - \\
7 & Informação \& Informação & 29 & $\mathrm{~A} 2$ & - \\
8 & Informação \& Sociedade: Estudos & 27 & $\mathrm{~A} 1$ & $\mathrm{Q} 3$ \\
9 & Revista P2P e INOVAÇÃO & 26 & $\mathrm{C}$ & - \\
10 & Revista Digital de Biblioteconomia \& Ciência da Informação & 20 & $\mathrm{~B} 1$ & $\mathrm{Q} 4$ \\
11 & Perspectivas em Gestão \& Conhecimento & 20 & $\mathrm{~B} 1$ & -
\end{tabular}

Fonte: elaborado pelos autores.

No presente estudo, observa-se que tal resultado tem como destaque a Reciis, com 492 trabalhos, ou seja, 43,2\% de todos os trabalhos recuperados. A Reciis surgiu em 2007 e tem em seu escopo publicações nas áreas de comunicação, informação e saúde coletiva. Está vinculada à Fiocruz e recebe financiamento do Instituto de Comunicação e Informação Científica e Tecnológica em Saúde da Fundação Oswaldo Cruz. A revista privilegia a publicação de textos com ênfase nas interfaces entre comunicação e saúde e entre informação e saúde.

Seu primeiro editor foi Carlos José Saldanha Machado, pesquisador da Fiocruz e que, junto da equipe editorial, procurou agregar as multidisciplinaridades, envolvendo a área de CI e saúde coletiva, incitando pesquisadores de diversas áreas a publicarem sobre o tema.

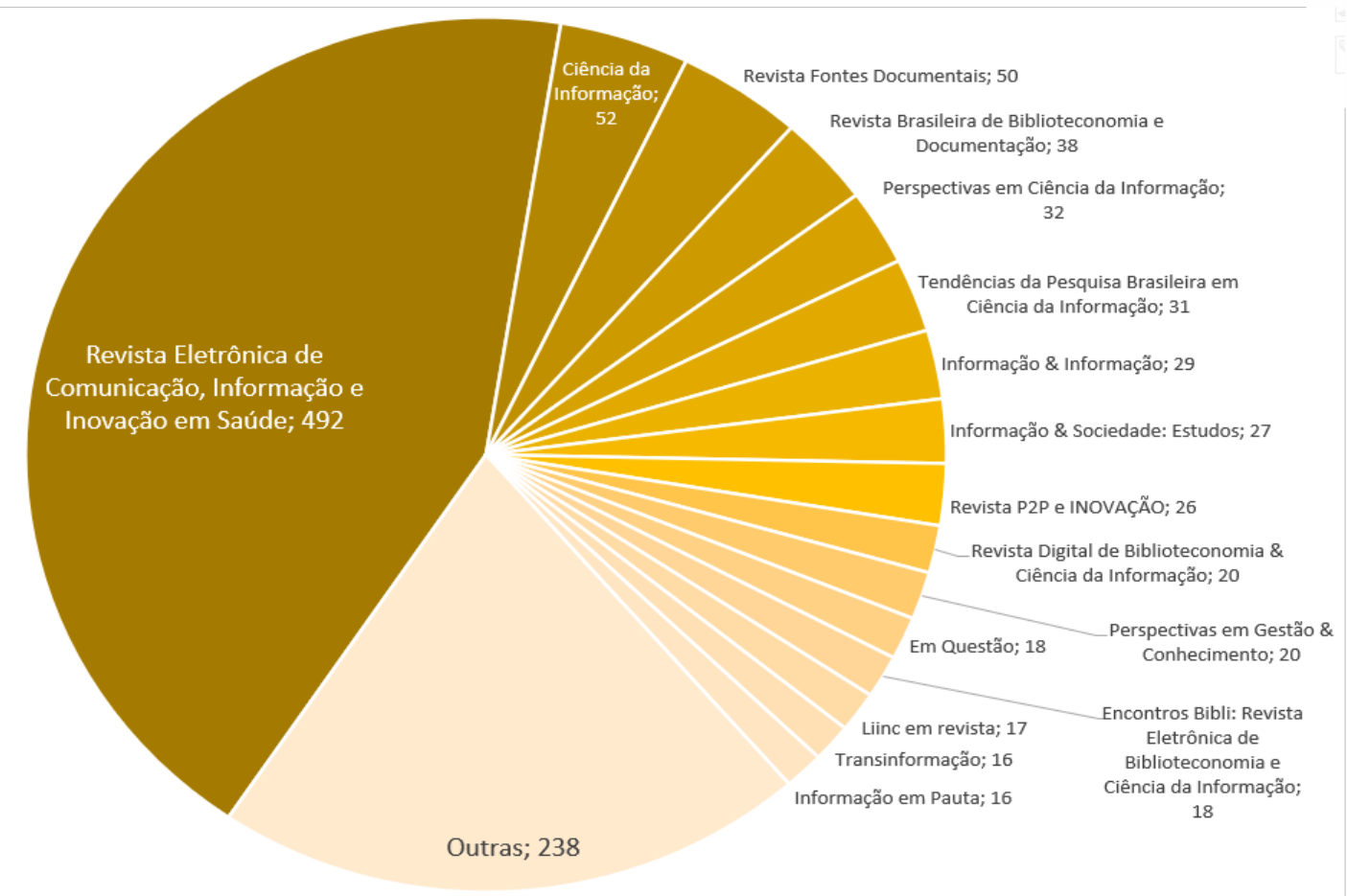

Figura 2 - Distribuição das publicações sobre as temáticas de saúde nas revistas de Cl brasileiras Fonte: elaboração dos autores. 
Alguns indicadores chamam a atenção, como os 17 trabalhos publicados na Revista Brasileira de Biblioteconomia e Documentação (RBBD), em 2017. Observou-se que 15 dos trabalhos identificados nesse ano foram publicados no número especial do XXVIII Congresso Brasileiro de Biblioteconomia, Documentação e Ciência da Informação (CBBD), envolvendo diversas práticas de bibliotecários em bibliotecas das áreas de saúde.

Outro destaque dessa variação está na Revista Ciência da Informação, que, em 2019, publicou 13 trabalhos em um único ano, sendo 12 deles publicados no número 3, e apenas um, no número 1 do volume 48 . O fascículo em questão, assim como a RBBD, publicou um suplemento especial dos trabalhos apresentados na $10^{\text {a }}$ Conferência Luso-Brasileira de Ciência Aberta (ConfOA).

Também é possível observar o crescimento da média de publicações do tema nas revistas. Se excluirmos a Reciis da análise, temos o total de 358 publicações nos últimos cinco anos e, considerando as 56 revistas ativas em CI ativas no Brasil, obtém-se uma média de 1,3 artigo por ano publicado em cada revista, um indicador ainda muito baixo ao comparar com a média de 39 artigos que cada revista publica anualmente.

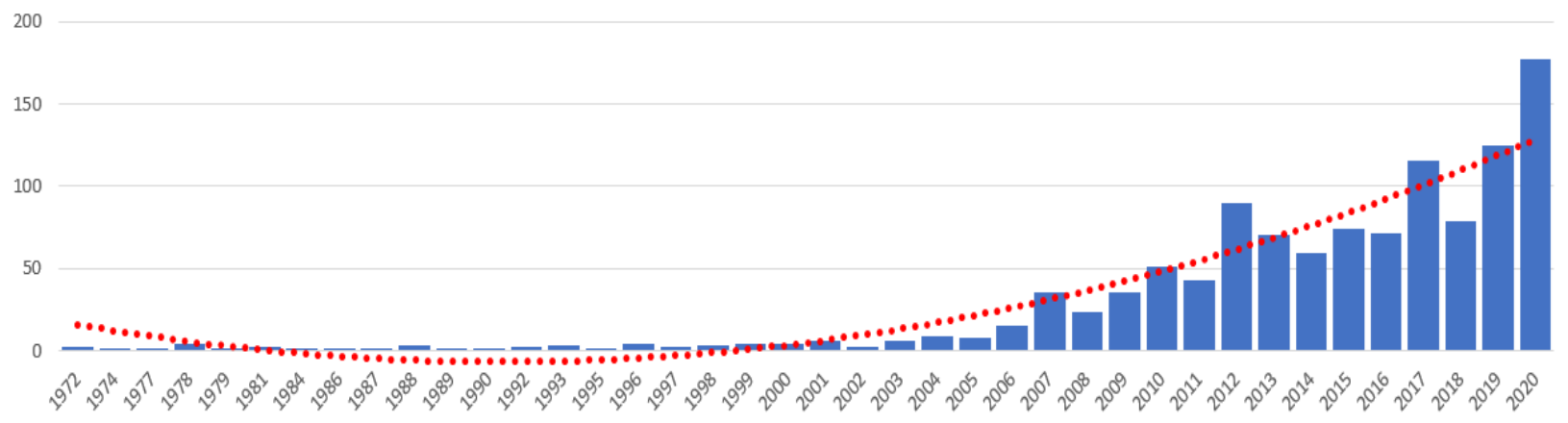

Figura 3 - Produção diacrônica do tema de saúde nas revistas de Cl brasileiras (1972-2020) Fonte: elaborado pelos autores.

Porém, ao visualizar a Figura 3, observa-se que a temática vem crescendo, ainda que de forma tímida, a partir de 2002. Na linha do fortalecimento da relação entre informação, comunicação e saúde, o PPGCII-Ibict/UFRJ está criando, em 2021, a Revista Asklepion, que tem como foco estudos e teorias da informação na área da saúde; organização do conhecimento e gestão da informação em saúde; e outras temáticas relacionadas às tecnologias de informação e comunicação, telemática, inteligência artificial relacionadas à saúde. 


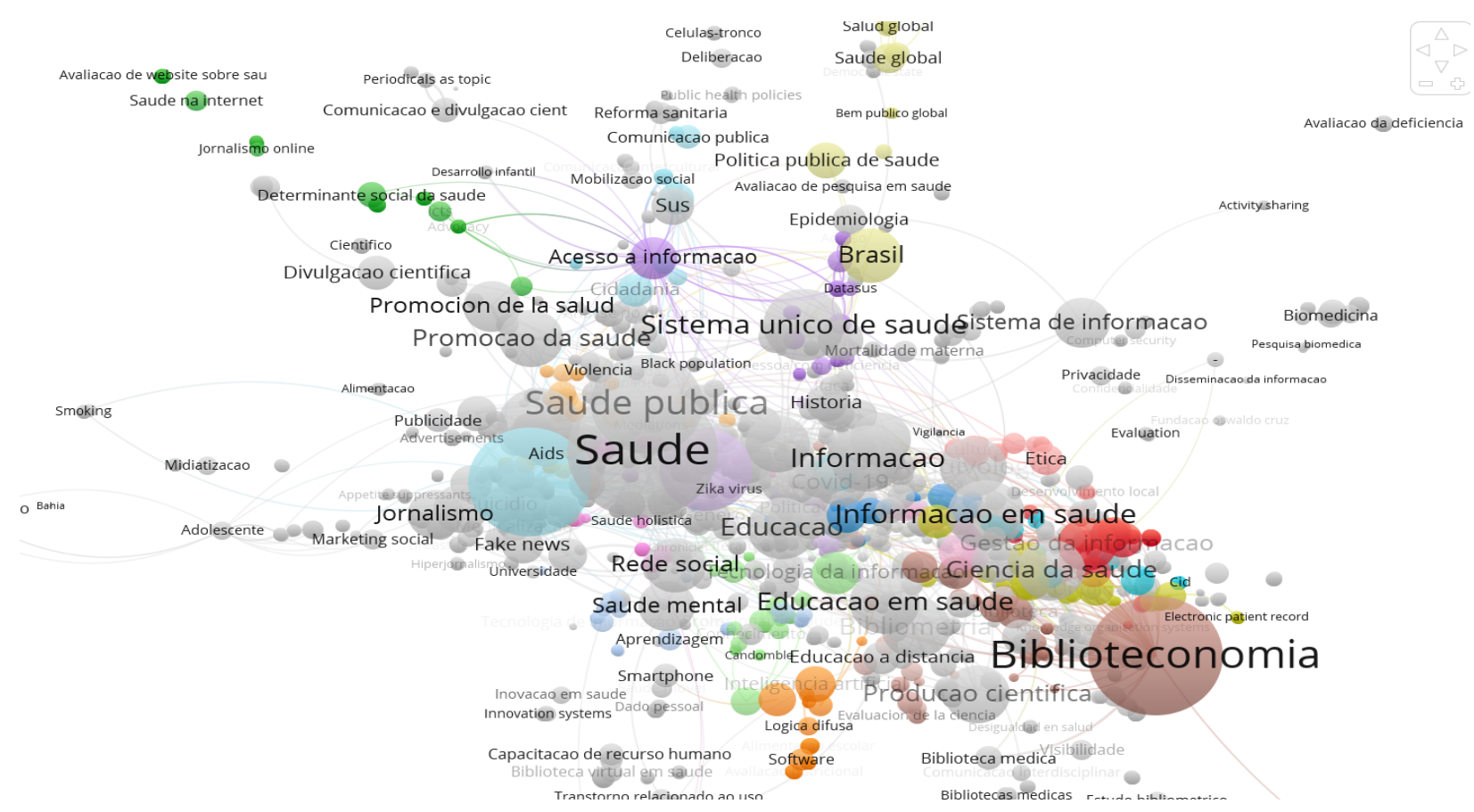

Figura 4 - Palavras-chave (português) e suas relações temáticas (1972-2020)

Fonte: elaborado pelos autores.

A pesquisa em pauta permite observar a prevalência de duas áreas: a primeira da biblioteconomia e a segunda de saúde pública; as temáticas que mais unem essas áreas se referem à informação, informação em saúde, educação, Sistema Único de Saúde e gestão da informação.

Outro destaque pode ser observado no termo 'acesso à informação', que está mais próximo da saúde pública e do Sistema Único de Saúde que da área de biblioteconomia.

Considerando que a área da saúde é composta por medicina, odontologia, farmácia, enfermagem, nutrição, fonoaudiologia, fisioterapia e terapia ocupacional e educação física, pode-se observar que não existe relação - ou há uma relação muito fraca - com a área de CI, sendo a saúde coletiva e/ou saúde pública que apresentam mais produção com a área de CI, influenciadas, principalmente, pelas pesquisas realizadas na Fiocruz/Icict.

\section{CONSIDERAÇÕES FINAIS}

A pesquisa aqui apresentada teve como objetivo geral analisar as aproximações da produção científica em ciências da saúde na área de CI, no Brasil, a partir dos artigos publicados em revistas brasileiras da área da CI e indexadas na Brapci, no período de 1972 a 2020.

Constatou-se que apenas 3,3\% dos documentos em CI indexados na base de dados Brapci abordam alguma questão da área de saúde, apesar de haver um GT específico para discussão da temática na ANCIB: o GT 11- Informação e Saúde.

Quanto ao objetivo de identificar os autores mais produtivos na temática, são pesquisadores vinculados a institutos de pesquisa, com destaque: Maria Cristina Soares Guimarães, pesquisadora da Fundação Oswaldo Cruz e professora permanente do Programa de Pós-Graduação em Informação e Comunicação em Saúde do Icict (PPGICS/Icict), sendo a mais produtiva, com 27 publicações entre 2006 e 2019. Além de outros pesquisadores ligados a institutos de pesquisa, também foram identificados docentes vinculados a programas de pós-graduação de universidades públicas, como os da Universidade Federal da Bahia, Universidade Federal do Rio de Janeiro e Universidade Federal de Minas Gerais. 
Em relação às formas de coautoria, constatou-se que, do total de publicações, 292 (25,6\%) foram de autoria única e 848 (74,4\%) de autoria múltipla, distribuídas em 367 (32,2\%) de dupla autoria, 231 (20,3\%) com autoria tripla, $124(10,9 \%)$ de autoria quádrupla, 126 (11,1\%) com cinco ou mais autores. A média de coautores por trabalhos foi de 1,98, demonstrando que a autoria múltipla identificada está de acordo com o padrão consolidado na área da saúde.

Já em relação às características dos periódicos utilizados para publicação, constatou-se que existe uma diversidade entre as revistas que publicam trabalhos na área de saúde na CI, mas, na identificação das mais produtivas, os artigos recuperados estavam distribuídos em 56 revistas brasileiras da área de CI. Entre elas, apenas 17 concentram mais de $80 \%$ dos artigos, sendo 43,2\% publicados pela Reciis e correspondentes a 492 trabalhos. Isso demonstra uma concentração em poucos títulos de revistas que têm a interseção com a área da saúde.

Quanto às temáticas identificadas a partir das palavras-chave, essas mostram a prevalência de duas áreas, a biblioteconomia e a saúde pública, e as temáticas que mais unem essas áreas, referentes à informação, informação em saúde, educação, Sistema Único de Saúde e gestão da informação. Outro destaque pode ser observado no termo "acesso à informação", que está mais próximo da saúde pública e do Sistema Único de Saúde que da área de biblioteconomia.

Conclui-se que a temática vem crescendo, ainda que de forma tímida, a partir de 2002, e sugere-se novos estudos sobre a presença de trabalhos relacionados à área da saúde na CI, em revistas estrangeiras, de forma a comparar os resultados com os obtidos nesta pesquisa.

\section{REFERÊNCIAS}

ARBOIT, Aline Elis; BUFREM, Leilah Santiago; LAZZAROTTO, Juliana. Configuração epistemológica na literatura periódica brasileira por meio de análise de citações (1972-2008). Perspectivas em Ciência da Informação, Belo Horizonte, v. 15, n. 2, p. 22-41, 2010. Disponível em: https://www.scielo.br/j/pci/a/D8dvPXVr CMPdhtzP9PygPcQ/?lang=pt\&format=pdf. Acesso em: 14 maio 2021.

BERAQUET, Vera Silvia Marao; CIOL, Renata. Atuação do bibliotecário em ambientes não tradicionais: o campo da saúde. Tendências da Pesquisa Brasileira em Ciência da Informação, [s. l.], v. 3, n. 1, p. $127-$ 137, 2010. Disponível em: http://revistas.ancib.org/index.php/tpbci/article/view/182. Acesso em: 23 set. 2021.

BETTIO, Maiara; ALVAREZ, Gonzalo Rubén; VANZ, Samile Andréa de Souza. Produção e colaboração científica da universidade federal de ciências da saúde de Porto Alegre. Informação \& Informação, Londrina, v. 22, n. 1, p. 88-110, 2017. DOI: http://dx.doi.org/10.5433/1981-8920.2017v22n1p88. Disponível em: http://hdl. handle.net/10183/163684. Acesso em: 6 maio 2021.

BICALHO, Lucinéia. Interações disciplinares presentes na pesquisa em ciência da informação.

Transinformação, Campinas, v. 23, n. 2, p. 113-126, 2011. DOI: https://www.doi.org/10.1590/S010337862011000200003. Disponível em: https://www.scielo.br/j/tinf/a/6fyFtNVBYcnmWQRpcMV9LTc/?lang=pt. Acesso em: 23 set. 2021.

BOCHNER, Rosany et al. Métricas contam a história e a trajetória da Revista Eletrônica de Comunicação, Informação e Inovação em Saúde - Reciis. Tendências da Pesquisa Brasileira em Ciência da Informação, [s. l.], v. 7, n. 2, 2014. Disponível em: http://hdl.handle.net/20.500.11959/brapci/119547. Acesso em: 14 maio 2021.

BOTOMÉ, Silvio Paulo. Pesquisa alienada e ensino alienante: o equívoco da extensão universitária. Petrópolis: Vozes,1996.

BUFREM, Leilah Santiago et al. Modelizando práticas para a socialização de informações: a construção de saberes no ensino superior. Perspectivas em Ciência da Informação, Belo Horizonte, v. 15, n. 2, p. 22-41, 2010. DOI: https://doi.org/10.1590/S1413-99362010000200003. Disponível em: https://www.scielo.br/j/pci/a/DH V9RQMtPKMrRRrdDMzMVvh/?lang=pt. Acesso em: 23 set. 2021.

CENTRE FOR SCIENCE AND TECHNOLOGY STUDIES (CWTS). Welcome to VOSviewer. Leiden: CWTS, 2021. Disponível em: https://www.vosviewer.coml. Acesso em: 20 jun. 2021. 
CENTRO LATINO-AMERICANO E DO CARIBE DE INFORMAÇÃO EM CIÊNCIAS DA SAÚDE (BIREME). 1967-2017: BIREME celebra 50 anos. Boletim BIREME, n. 5, 24 fev. 2017. Disponível em: https://boletin. bireme.org/pt/2017/02/24/1967-2017-bireme-celebra-50-anos/. Acesso em 30 jul. 2021.

COORDENAÇÃO DE APERFEIÇOAMENTO DE PESSOAL DE NÍVEL SUPERIOR (CAPES). Tabela de áreas de conhecimento/avaliação. Brasília, DF: Capes, 2017. Disponível em: https://www.gov.br/capes/pt-br/ acesso-a-informacao/acoes-e-programas/avaliacao/instrumentos/documentos-de-apoio-1/tabela-de-areas-deconhecimento-avaliacao. Acesso em: 3 maio 2021.

FUNDAÇÃO OSWALDO CRUZ (FIOCRUZ). Instituto de Comunicação e Informação Científica e Tecnológica em Saúde (ICICT). Informação e comunicação científica para o SUS: relatório de atividades 2011-2012. Rio de Janeiro: Icict, 2013. Disponível em: https://www.icict.fiocruz.br/sites/www.icict.fiocruz.br/files/relatorio-finalgrafica_Mauro_optm.pdf. Acesso em: 24 set. 2021.

GABRIEL JUNIOR, Rene Faustino. Panorama dos 45 anos das revistas de ciência da informação no Brasil: um estudo na Brapci. In: ENCONTRO NACIONAL DE PESQUISA EM CIÊNCIA DA INFORMAÇÃO,18., 2017, Marília. Anais [...]. Marília: Unesp, 2017. Disponível em: http://hdl.handle.net/20.500.11959/brapci/105548. Acesso em: 12 maio 2021.

GARCIA, João Paulo dos Santos; ARAUJO, Nelma Camêlo de; SOUZA, Edivanio Duarte de. Informação, gênero e sexualidade: uma análise da comunicação científica do grupo de trabalho "informação \& saúde" Enancib. Convergência em Ciência da Informação, São Cristóvão, v. 2 n. 2, p. 83-104, 2019. DOI: https:// doi.org/10.33467/conci.v2i2.12837. Disponível em: https://seer.ufs.br/index.php/conci/article/view/12837/10197. Acesso em: 3 maio 2021.

GLÄNZEL, Wolfgang. National characteristics in international scientific coauthorship relations. Scientometrics, Amsterdã, v. 51, n.1, p. 69-115, 2001. DOI: https://doi.org/10.1023/A:1010512628145. Disponível em: https:// link.springer.com/article/10.1023/A:1010512628145. Acesso em: 4 maio 2021.

GRACIO, Maria Cláudia Cabrini; OLIVEIRA, Ely Francina Tannuri de. A inserção e o impacto internacional da pesquisa brasileira em "estudos métricos": uma análise na base Scopus. Tendências da Pesquisa Brasileira em Ciência da Informação, [s. I.], v. 5, n. 1, p. 1-19, 2012. Disponível em: http://inseer.ibict.br/ancib/index.php/ tpbci/article/view/71/113. Acesso em: 1 ago. 2021.

GUIMARÃES, José Augusto Chaves. Organização do conhecimento: passado, presente e futuro sob a perspectiva da ISKO. Informação \& Informação, Londrina, v. 22, n. 2, p. 84-98, 2017. DOI: http://dx.doi. org/10.5433/1981-8920.2017v22n2p84. Disponivel em: http://www.uel.br/revistas/uel/index.php/informacaol article/view/31443/21993. Acesso em: 2 ago. 2021.

HILÁRIO, Carla Mara; GRÁCIO, Maria Cláudia Cabrini; GUIMARÃES, José Augusto Chaves. Aspectos éticos da coautoria em publicações científicas. Em Questão, Porto Alegre, v. 24, n. 2, p. 12-36, 2018. DOI: https:// doi.org/10.19132/1808-5245242.12-36. Disponível em: https://seer.ufrgs.br/EmQuestao/article/view/76312/0. Acesso em: 3 ago. 2021.

HILÁRIO, Carla Mara; LAZZAROTO, Juliana. Indicadores de colaboração científica: aspectos éticos, práticos e formas de mensuração. In: GRÁCIO, Maria Cláudia Cabrini et al. (org.). Tópicos da bibliometria para bibliotecários universitários. Marília: Oficina Universitária, 2020. p. 71-92.

KATZ, J. Sylvan; MARTIN, Ben R. What is research collaboration? Research Policy, Amsterdã, v. 26, n. 1, p. 1-18, 1997. DOI: https://doi.org/10.1016/S0048-7333(96)00917-1. Disponível em: https://www.sciencedirect. com/science/article/abs/pii/S00487333396009171. Acesso em: 4 maio 2021.

LAZZAROTTO, Juliana. Dimensões da pesquisa brasileira no interdomínio dos estudos métricos da informação em medicina. 2017. 201 f. Tese (Doutorado em Ciência da Informação) - Faculdade de Filosofia e Ciências, Universidade Estadual Paulista, Marília, São Paulo, 2017.

LOURENÇO, Cíntia de Azevedo. A automaç̧ão em bibliotecas: análise da produção via Biblioinfo (1986/1994). In: WITTER, Geraldina Porto (org.). Produção científica. Campinas: Átomo, 1997. p. 25-40.

MAIA, Maria de Fátima Santos. A produção e o uso de informação em saúde: estudo bibliométrico da área de epidemiologia. 2006. 119 f. Dissertação (Mestrado em Comunicação e Informação) - Faculdade de Biblioteconomia e Comunicação, Universidade Federal do Rio Grande do Sul, Porto Alegre, 2006. 
MATTOS, Ana Maria; JOB, Ivone. A produção científica brasileira no periódico Scientometrics de 1978 até 2006. Encontros Bibli: Revista Eletrônica de Biblioteconomia e Ciência da Informação, Florianópolis, n. 26, p. 47-61, 2008. DOI: https://doi.org/10.5007/1518-2924.2008v13n26p47. Disponível em: https://periodicos. ufsc.br/index.php/eb/article/view/1518-2924.2008v13n26p47/6934. Acesso em: 3 ago. 2021.

MEADOWS, Arthur Jack. A comunicação científica. Brasília, DF: Briquet de Lemos/Livros, 1999.

MOREIRA, Fábio Mosso; VALENTIM, Marta Lígia Pomim; SANTANA, Ricardo César Gonçalves. A interdisciplinaridade da ciência da informação e suas contribuições no estudo do compartilhamento de dados governamentais na internet. Em Questão, Porto Alegre, v. 24, n. 2, p. 300-329, 2018. DOI: https:// doi.org/10.19132/1808-5245242.300-329. Disponível em: https://seer.ufrgs.br/index.php/EmQuestao/article/ view/75308. Acesso em: 23 set. 2021.

MUELLER, Suzana Pinheiro Machado. A publicação da ciência: áreas científicas e seus canais preferenciais. DataGramaZero: Revista de Ciência da Informação, [s, I.], v. 6, n.1, p.1-12, 2005. Disponível em: https:// repositorio.unb.br/bitstream/10482/980/2/ARTIGO PublicacaoCiencia.pdf. Acesso em: 2 maio 2021.

MUELLER, Suzana Pinheiro Machado; PECEGUEIRO, Cláudia Maria Pinho de Abreu. O periódico Ciência da Informação na década de 90: um retrato da área refletido em seus artigos. Ciência da Informação, Brasília, DF, v. 30, n. 2, p. 47-63, 2001. Disponível em: http://revista.ibict.br/ciinf/article/view/924/961. Acesso em: 30 maio 2021.

NASCIMENTO, Dandara Souza Araújo et al. Projeções exponenciais da ciência brasileira: modelos e análises quantitativas da produção científica nacional publicada nos últimos 30 anos. Informação \& Informação, Londrina, v. 26, n. 1, p. 53-73, 2021. DOI: http://dx.doi.org/10.5433/1981-8920.2021v26n1p53. Disponível em: http://www.uel.br/revistas/uel/index.php/informacao/article/view/40628. Acesso em: 2 maio 2021.

NEVES, Bruno; FERREIRA, Carla. Caracterização da produção científica portuguesa em ciência da informação disponibilizada em acesso aberto no e-LIS. Cadernos BAD, Lisboa, n. 2, p. 95-98, 2014. Disponível em: http://hdl.handle.net/20.500.11959/brapci/68379. Acesso em: 6 maio 2021.

PRUDENCIO, Dayanne da Silva; BIOLCHINI, Jorge Calmon de Almeida. Informação e saúde nos currículos dos cursos de biblioteconomia do Brasil. Tendências da Pesquisa Brasileira em Ciência da Informação, [s. I.], v. 11, n. 1, p. 1-22, 2018. Disponível em: https://revistas.ancib.org/index.php/tpbci/ article/view/503/483. Acesso em: 3 ago. 2021.

PRUDENCIO, Dayanne da Silva; BIOLCHINI, Jorge Calmon de Almeida. Temática informação e saúde na pós-graduação em ciência da informação. In: ENCONTRO NACIONAL DE PESQUISA EM CIÊNCIA DA INFORMAÇÃO, 18., 2017, Marília. Anais [...]. [S. I.]: Enancib, 2017. Disponível em: http://hdl.handle. net/20.500.11959/brapci/105330. Acesso em: 03 maio 2021.

QUEIROZ, Daniela Gralha de Caneda. A autoria única nos artigos de periódicos das áreas de informação no Brasil (2009-2017). 2020. 283 f. Tese (Doutorado em Ciência da Informação) Programa de Pós-graduação em Ciência da Informação, Universidade de Brasília, Brasília, DF, 2020.

SIDONE, Otávio José Guerci; HADDAD, Eduardo Amaral; MENA-CHALCO, Jesús Pascual. A ciência nas regiões brasileiras: evolução da produção e das redes de colaboração científica. Transinformação, Campinas, v. 28, n. 1, p. 15-31, 2016. DOI: https://doi.org/10.1590/2318-08892016002800002. Disponível em: https://www.scielo.br/pdf/tinf/v28n1/0103-3786-tinf-28-01-00015.pdf. Acesso em: 4 maio 2021.

SOBRAL, Natanael Vitor et al. Produção científica colaborativa na área da saúde tropical: uma análise da rede de colaboração do Programa de Pós-Graduação em Medicina Tropical da Universidade Federal de Pernambuco. Revista Eletrônica de Comunicação, Informação e Inovação em Saúde, Rio de Janeiro, v. 10, n. 1, p. 1-15, 2016. DOI: https://doi.org/10.29397/reciis.v10i1.1025. Disponível em: https:// www.reciis.icict.fiocruz.br/index.php/reciis/article/view/1025. Acesso em: 10 maio 2021.

SONNENWALD, Diane H. Scientific Collaboration. Annual Review of Information Science and Technology, New York, v. 42, n. 1, p. 643-681, 2008. DOI: https://doi.org/10.1002/aris.2007.1440410121. Disponível em: https://asistdl.onlinelibrary.wiley.com/doi/10.1002/aris.2007.1440410121. Acesso em: 29 set. 2021.

SMIT, Johanna Wilhelmina. A pesquisa na área de ciência da informação. Transinformação, Campinas, v. 14, n. 1, p. 25-28, 2002. DOI: http://dx.doi.org/10.1590/S0103-37862002000100003. Disponível em: https://www.scielo.br/j/tinf/a/sPdxSKgywKtFV5nj9L5mgXv/?lang=pt. Acesso em: 23 set. 2021. 
TARGINO, Maria das Graças. Informação em saúde: potencialidades e limitações. Informação \& Informação, Londrina, v. 14, n. 1, p. 52-81, 2009. Disponível em: https://www.brapci.inf.br/ repositorio/2010/05/pdf 388191884b 0010347.pdf. Acesso em: 1 ago. 2021.

VANZ, Samile Andrea de Souza. As redes de colaboração científica no Brasil (2004-2006). 2009. 204 p. Tese (Doutorado em Comunicação e Informação) - Faculdade de Biblioteconomia e Comunicação, Universidade Federal do Rio Grande do Sul, Porto Alegre, 2009.

VANZ, Samile Andrea de Souza; STUMPF, Ida Regina Chittó. Colaboração científica: revisão teóricoconceitual. Perspectivas em Ciência da Informação, Belo Horizonte, v. 15, n. 2, p. 42-55, 2010. Disponível em: http://portaldeperiodicos.eci.ufmg.br/index.php/pci/article/view/1105/731. Acesso em: 2 maio 2021. 